\title{
Evaluación de daño de gorgojos en poblaciones de alfalfa (Medicago sativa L.) con alto número de raíces laterales
}

\author{
Odorizzi, A. S.; V. Arolfo y D. H. Basigalup
}

\begin{abstract}
RESUMEN
El aumento del número de raíces laterales a través del mejoramiento puede ser importante para reducir el daño provocado por el complejo de gorgojos de la alfalfa. El objetivo fue evaluar, en cuatro ambientes (siembras de otoño y primavera con y sin riego), el comportamiento de 10 poblaciones seleccionadas por alto número de raíces laterales. Los caracteres evaluados fueron: categoría (Cat) de daño de gorgojos (de $1=\sin$ daño a $5=$ daño severo), rendimiento promedio de forraje (BP), número de raíces secundarias (NRLR) y diámetro de raíces laterales (DRLR). Las poblaciones s755, s545 y s614 presentaron los mayores valores de DRLR y NRLR, el menor daño de gorgojos (Cat $2+3)$ y la mayor variabilidad para los caracteres estudiados. Las poblaciones s 545 y s616 exhibieron el mayor DRLR y el menor NRLR, respectivamente. Las condiciones de riego propiciaron un menor daño, y fueron menos afectadas en estos ambientes las poblaciones s545, s614 y s617; por el contrario, las poblaciones s461, s755, s463 resultaron las más afectadas. Los daños más severos (Cat $4+5)$ se observaron en secano y las poblaciones 5618 y s616 fueron las más afectadas; sólo bajo estas condiciones el mayor daño se correlacionó con menor BP.
\end{abstract}

Palabras clave: alfalfa, raíz ramificada, daño de gorgojos.

Odorizzi, A. S.; V. Arolfo and D. H. Basigalup, 2011. Evaluation of root curculio/weevil damage in alfalfa (Medicago sativa L.) populations with large numbers of lateral roots. Agriscientia XXVIII (2): 119-126

\section{SUMMARY}

Increasing the number of lateral roots through breeding may be important to reduce the damage caused by the root curculio complex in alfalfa in Argentina. The objective was to evaluate the performance of ten alfalfa experimental populations selected for their large number of lateral roots under four environmental conditions (fall and spring planting with or without irrigation). The evaluated traits were curculio damage (Categories (Cat) $1=$ no damage to $5=$ very severe damage), dry matter yield (BP), number of secondary roots 
(NRLR), and diameter of lateral roots (DRLR). Populations s755, s545 and s614 had the highest values for DRLR and NRLR, the least damage from weevil (Cat $2+3$ ), and the largest variability (mean square) for all the traits. Populations s545 and s616 exhibited the highest DRLR and the lowest NRLR, respectively. Irrigation conditions led to lesser curculio damage, with populations s545, s614 and s617 being the least affected, and populations s461, s755 and s463, the most affected. The highest damage (Cat $4+5)$ was observed under rainfed conditions (dryland), with populations s618 and s616 as the most affected by the root curculio complex. Only under these conditions the highest curculio damage was correlated with lowest BP.

Keywords: alfalfa, lateral roots, root weevil damage.

A. S. Odorizzi, V. Arolfo y D. H. Basigalup. INTA - EEA Manfredi. Ruta Nac. No 9 km. 636, (5988) Manfredi, Córdoba, Argentina. Correspondencia a A. Odorizzi: aodorizzi@manfredi.inta.gov.ar

\section{INTRODUCCIÓN}

El cultivo de alfalfa (Medicago sativa L.) es uno de los pilares de la producción agropecuaria argentina, tanto por sus altos rendimientos en cantidad y calidad de forraje como por su efecto mejorador de suelos y su amplia adaptación a regiones agroecológicas muy diversas. La perennidad de la alfalfa y la densidad de plantas del cultivo generan un ambiente favorable para la proliferación de enfermedades y de muchas especies de ácaros e insectos. La prevención y el control de organismos dañinos adquieren una importancia fundamental para el mantenimiento de lotes productivos (Sulc y Rodes, 1997). En la Argentina, del 80 al $90 \%$ del daño de plagas a la alfalfa es provocado por cuatro grupos de insectos: orugas desfoliadoras, orugas cortadoras, pulgones y gorgojos (Aragón e Imwinkelried, 2007). Los gorgojos (Coleoptera: Curculionidae), forman un verdadero complejo con alrededor de 22 especies pertenecientes en su mayoría al género Naupactus. El estadio larval transcurre dentro del suelo. Debido a la superposición de generaciones de las distintas especies presentes, pueden encontrarse larvas de varios estadios de desarrollo durante el año, las que provocan severos daños a las raíces de alfalfa de las cuales se alimentan. Esto disminuye la productividad y la persistencia del cultivo (Imwinkelried et al., 1992) y en algunos casos llega a causar la muerte indirecta de plantas al facilitar la entrada de microorganismos patógenos (Aragón, 1990). Las consecuencias pueden ser fatales en plántulas o plantas jóvenes, ya que las lesiones en la raíz principal pueden provocar la destrucción de ese sistema radical incipiente (Parodi, 1964). Ataques severos ocasionan la mortandad de plantas en manchones, que se incrementan hasta la pérdida total de los lotes afectados (Itria, 1969). El manejo del complejo de gorgojos constituye uno de los mayores desafíos para el control de plagas, dados su hábito de alimentación polífago y los distintos picos poblacionales a lo largo del año de las especies que integran el complejo (Harcourt et al., 1986). Además, los estadios preimaginales y los huevos se hallan protegidos dentro de la tierra, ya sea entre terrones o cubiertos con diferentes sustancias, lo que dificulta considerablemente su control químico. No existen métodos de control eficientes de larvas y pueden registrarse daños aun cuando la semilla sea tratada con insecticidas (Gassen, 1996). La ausencia de fuentes de resistencia genética y de adecuados protocolos de selección invalida el empleo de métodos tradicionales de mejoramiento, haciendo que no haya antecedentes de variedades resistentes (Todd, 1968; De Durana, 1981; Bariggi et al., 1986; Basigalup e Hijano, 1995).

Una posible forma de atenuar sus daños sería contar con genotipos que posean un sistema radical ramificado o con alto número de raíces laterales, como forma de ofrecer mayores porciones de tejido radical que puedan escapar al ataque de los gorgojos. Otra forma sería disponer de plantas capaces de regenerar sus raíces cuando éstas son cortadas o destruidas por la plaga.

La selección fenotípica recurrente (SFR) es un método de mejoramiento muy eficiente que posibilita, en un corto intervalo generacional y con 
un sistema cíclico de selección de individuos, una gran intensidad de selección para incrementar la frecuencia de alelos favorables (Dudley et al., 1963). Desde hace algunos años, la Estación Experimental Agropecuaria (EEA) Manfredi del Instituto Nacional de Tecnología Agropecuaria (INTA), ha reunido una importante colección con materiales de diferente grado de reposo invernal (GRI 4 a 9) y de muy diferentes orígenes. Ese germoplasma fue sometido a SFR por alto número de raíces laterales o por raíces ramificadas. Los genotipos seleccionados fueron luego combinados en poblaciones experimentales.

El objetivo del presente trabajo fue evaluar, bajo diferentes condiciones ambientales, el comportamiento de las poblaciones experimentales antes mencionadas frente al daño provocado por gorgojos.

\section{MATERIALES Y MÉTODOS}

El material vegetal a evaluar estuvo compuesto por 10 poblaciones experimentales (P) desarrolladas en la EEA Manfredi por el programa de mejoramiento genético de alfalfa a partir de un grupo de accesiones provenientes de la colección estadounidense de medicagos perennes. Después de cuatro ciclos de SFR por alto número de raíces laterales y/o sistema radical ramificado, se obtuvieron las siguientes poblaciones experimentales: s461 (GRI 6), s463 (GRI 8), s545 (GRI 6), s614 (GRI 7), s616 (GRI 9), s617 (GRI 7), s618 (GRI 9), s634 (GRI 8), s755 (GRI 7) y s923 (GRI 8).

Los ensayos de evaluación se realizaron durante 2004 en un lote de la EEA Manfredi con antecedentes de alta infestación de gorgojos y caracterizado como Haplustol éntico (Serie Oncativo), profundo, bien drenado, ubicado en la región pampeana semiárida, con $744 \mathrm{~mm}$ de precipitación media anual. Se utilizó un diseño experimental en bloques completos aleatorizados (DBCA) con tres repeticiones y una densidad de siembra de 350-400 semillas viables $\mathrm{m}^{-2}$. A fin de evaluar los materiales bajo diferentes condiciones, se definieron cuatro ambientes combinando dos fechas de siembra (otoño y primavera) y dos regímenes hídricos (secano y riego). De esta manera, se implantó un ensayo dentro de cada uno de los ambientes (A) creados: 1- otoño en secano (otosecano); 2- otoño con riego (otoriego); 3primavera en secano (primsecano); y 4- primavera con riego (primriego). Como testigos se utilizaron los cultivares comerciales 5683 (GRI 6), Gala (GRI 7) y Monarca SP INTA (GRI 8), todos con sistema radical predominantemente pivotante, y una combinación (pool) de GRI 8 que incluyó algunas accesiones originales de las cuales derivaron las poblaciones a evaluar. Las parcelas tuvieron una dimensión de $3 \times 5 \mathrm{~m}$ con una distancia entre hileras de 0,20 m.

Cumplido el primer año en cada ambiente, se extrajeron todas las plantas de una subparcela de 1 $\times 1 \times 0,80 \mathrm{~m}$ en cada unidad experimental. Del total de plantas extraídas y lavadas se tomó una muestra al azar de 30 plantas y se evaluó: a) categoría de daño de gorgojos (Cat) según la siguiente escala visual: 1- sin daño; 2- daño leve, con muy pocas canaletas visibles; 3 - daño moderado, con canaletas visibles pero poco profundas; 4- daño severo, con canaletas profundas; y 5- daño muy severo, con abundantes canaletas profundas, raíces cortadas o muy destruidas y presencia de infección secundaria en las áreas afectadas; b) número de raíces laterales ó ramificadas (rNRLR), por visualización directa (para el análisis estadístico, al ser una variable de conteo, se transformaron los datos a raíz cuadrada); c) diámetro de raíces laterales ó ramificadas (DRLR), medido en mm con un calibre en el punto inmediato de inserción en la raíz pivotante. Para la evaluación de la biomasa aérea (BP), se definió dentro de cada unidad experimental otra subparcela fija de $1 \times 5 \mathrm{~m}$, en la que se cortó y pesó todo el forraje producido cada vez que se alcanzaba el 10\% de floración o cuando los rebrotes desde la corona medían aproximadamente 0,05 m. La producción se expresó en materia seca (MS).

Para cada variable evaluada, se realizó un análisis de la varianza (ANAVA) para un modelo factorial con repeticiones anidadas en ambiente. También se realizó un análisis de componentes principales (ACP) con todas las variables, a fin de explicar el comportamiento de las poblaciones a través de los ambientes. Se interpretó visualmente en cada uno de los gráficos biplot la asociación entre las variables con base en la longitud del vector. La medida de asociación entre las variables se estimó a través del coeficiente de correlación de Pearson ( $r$ ). Los análisis estadísticos se realizaron con el software Info-Gen/P 2006i.1 Versión 1.0 (Balzarini y Di Rienzo, 2004).

\section{RESULTADOS Y DISCUSIÓN}

En el ACP que se muestra en la Figura 1, utilizado para reducir la dimensionalidad del conjunto de datos generados y poder jerarquizar por importancia las causas de la variabilidad, se observa que a nivel de la CP1, que es la que 


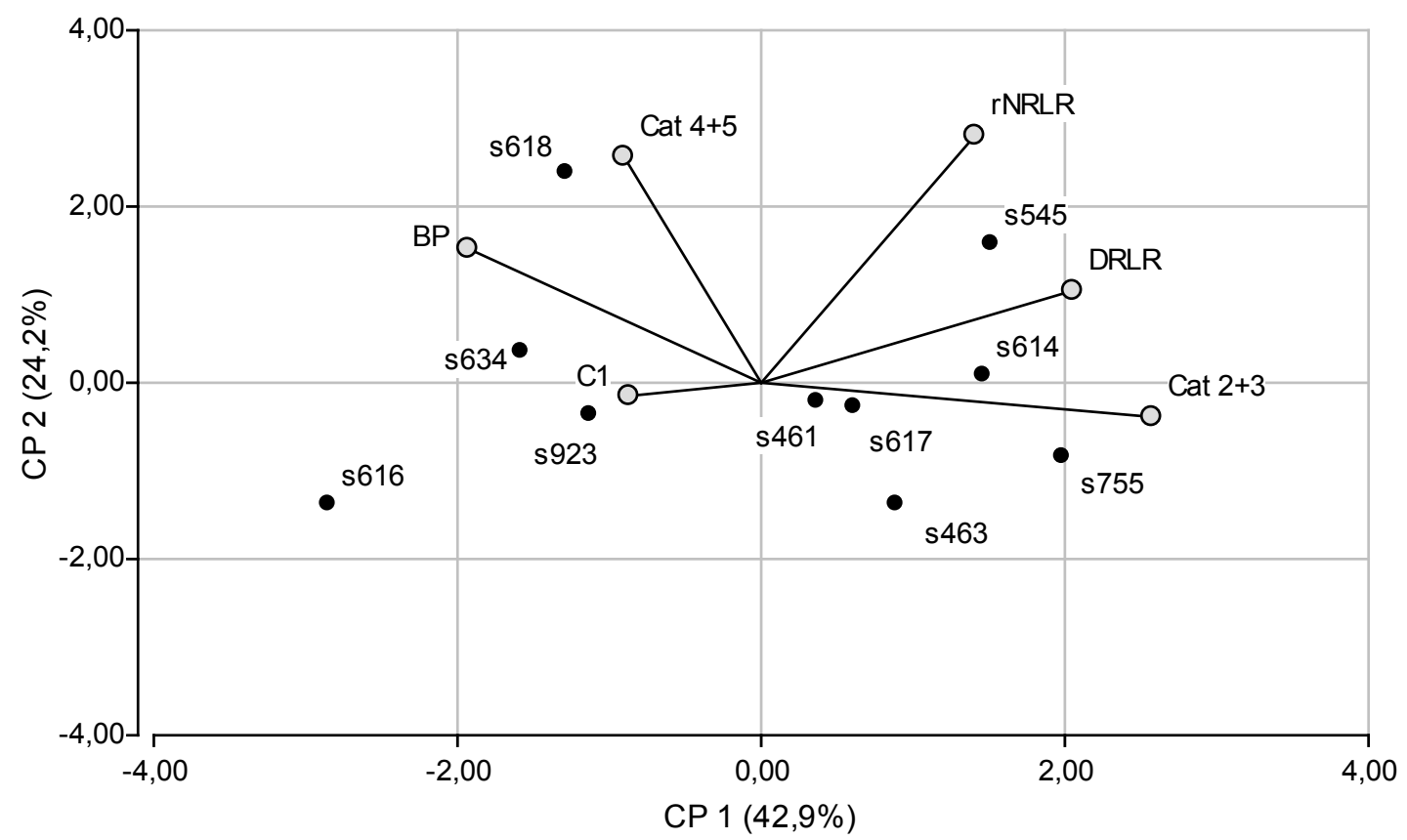

Figura 1. Plano biplot generado por el análisis de componentes principales para las categorías de daño de gorgojos, variables de raíz y biomasa aérea entre la media de las poblaciones y los cuatro ambientes definidos (otoño en secano, otoño bajo riego, primavera en secano y primavera bajo riego). Ref.: Cat: categoría de daño de gorgojos, BP: biomasa promedio, DRLR: diámetro de raíces laterales o ramificadas y rNRLR: raíz del número de raíces laterales o ramificadas.

explica por sí sola el $42,9 \%$ de la varianza total, las variables DRLR, rNRLR y daño moderado (Cat $2+3)$ exhibieron la mayor inercia y tendieron a correlacionarse positivamente. Las poblaciones s755, s545 y s614 presentaron los mayores valores para esos caracteres y también la mayor variabilidad. Esto es coincidente con un trabajo previo de Odorizzi et al. (2008), en el que se concluyó que la SFR por alto número de raíces laterales o por sistema radical ramificado había sido muy eficiente en el desarrollo de las poblaciones s545 y s614, que se destacaron para esos caracteres en todos los ambientes incluidos en ese estudio. Por otro lado, es interesante señalar que estas tres poblaciones son de reposo invernal intermedio y que se diferenciaron grandemente por NRLR de las poblaciones s923, s616, s618 y s634, que no poseen reposo invernal; en ese contexto, s616 fue la más extrema ya que presentó un sistema radicular pivotante sin raíces laterales y el mayor diámetro de raíz pivotante. Según Johnson et al. (1998), las alfalfas sin reposo presentan mayoritariamente una raíz pivotante sin ramificaciones, en tanto que las de reposo invernal intermedio tienen una raíz pivotante de crecimiento más bien determinado y un mayor número de raíces laterales. Por su parte, las alfalfas de mayor reposo $(G R I \leq 5)$ suelen presentar el mayor número de raíces laterales, en relación inversa con su GRI.

El ANAVA realizado para las variables en estudio (Tabla 1) no detectó interacción $\mathrm{P}^{\star} \mathrm{A}$ para las categorías de daño de gorgojos y para las

Tabla 1. Análisis de la varianza para las categorías de daño provocado por larvas de gorgojos y las variables de raíz y biomasa aérea entre poblaciones y testigos para todos los ambientes.

\begin{tabular}{|c|c|c|c|}
\hline \multirow[b]{2}{*}{ Variables $^{(t)}$} & \multicolumn{3}{|c|}{ Cuadrado medio } \\
\hline & $P^{*} \mathbf{A}$ & Población (P) & Ambiente (A) \\
\hline Cat 1 & $400,92^{n s}$ & $612,26^{\mathrm{ns}}$ & $17602,89^{\star \star}$ \\
\hline Cat 2 & $174,02^{\mathrm{ns}}$ & $167,35^{\text {ns }}$ & $258,86^{\text {ns }}$ \\
\hline Cat 3 & $135,61^{\mathrm{ns}}$ & $317,94^{*}$ & $4430,95^{\star *}$ \\
\hline Cat 4 & $131,49^{\mathrm{ns}}$ & $119,75^{\mathrm{ns}}$ & $1775,76^{\star \star}$ \\
\hline Cat 5 & $28,16^{\text {ns }}$ & $45,64^{*}$ & $461,67^{\star \star}$ \\
\hline Cat $2+3$ & $322,40^{\mathrm{ns}}$ & $558,69^{n s}$ & $8627,66^{\star *}$ \\
\hline Cat $4+5$ & $204,85^{\text {ns }}$ & $210,24^{n s}$ & $3599,88^{* *}$ \\
\hline BP & $2702^{*}$ & $1958^{\text {ns }}$ & $194281^{\star *}$ \\
\hline DRLR & $0,001^{\mathrm{ns}}$ & $0,002^{*}$ & $2,58^{*}$ \\
\hline rNRLR & $0,01^{\mathrm{ns}}$ & $0,02^{\star \star}$ & $0,04^{*}$ \\
\hline
\end{tabular}

${ }^{\left({ }^{+} C\right.}$ Cat: categoría de daño de gorgojos, BP: biomasa promedio, DRLR: diámetro de raíces laterales o ramificadas y rNRLR: raíz del número de raíces laterales o ramificadas.

${ }^{*},{ }^{* \star}$, ns: significativo a los niveles de probabilidad 0,05, 0,01 y no significativo, respectivamente. 
variables de raíz ramificada, lo que indica que las diferencias entre las poblaciones y testigos evaluados se mantuvieron a través de los diferentes ambientes. Por el contrario, la variable BP tuvo una interacción $P^{\star} A$ estadísticamente significativa, lo que indica influencia del ambiente (básicamente riego) en su expresión.

Respecto del daño de gorgojos, se detectaron diferencias estadísticamente significativas entre los ambientes (con excepción de Cat 2) pero no entre las poblaciones y los testigos, excepto para las Cat 3 y $5(p>0,05)$. Tanto para la variable DRLR como para rNRLR, las mayores diferencias se encontraron entre las poblaciones s545 y s616, de mayor y menor DRLR y rNRLR, respectivamente.

El efecto del ambiente sobre las categorías de daño de gorgojos se analizó a través de un modelo GGE biplot. En la Figura 2 se muestra el análisis para la Cat 1 (sin daño), donde se aprecia que la inclusión de riego propició un menor ataque de gorgojos (Figura 2). Esto es coincidente con uno de los métodos culturales más recomendados para prevenir el daño de estos insectos, que consiste en mantener un alto nivel de humedad en el suelo (Thomason et al., 1985). Con base en la longitud del vector determinado para cada ambiente, y a que los extremos que definen el polígono envolvente del biplot están dados por las poblaciones de comportamiento extremo, también se concluye de la Figura 2 que en los ambientes bajo riego las poblaciones s545, s614 y s617, al presentar la mayor cantidad de plantas ubicadas en la Cat 1, fueron las menos afectadas por los gorgojos. En igual sentido, el análisis para grados de daño leve a moderado (Cat $2+3$ ) que se exhibe en la Figura 3 muestra que los ambientes irrigados concentraron más daños moderados que los de secano; las poblaciones s461, s755, s463 y el testigo Monarca SP INTA fueron las que presentaron mayor cantidad de plantas en las Cat $2+3$. Finalmente, y como era de esperar, los grados de daño alto a extremo (Cat 4+5) se manifestaron con mayor intensidad en los ambientes en secano, más propicios para el desarrollo de la plaga (Figura 4). En estas condiciones, las poblaciones s618 y s616 exhibieron los mayores niveles de afectación. Es de hacer notar que s616 es la población de características radiculares más similar a los testigos, con raíz pivotante y menor número de raíces secundarias. Según Berry (1947) los daños ocasionados por gorgojos aumentan en épocas de sequía. También Altier y Alzugaray (1990) encontraron mayores daños en ensayos parcelarios de alfalfa bajo condiciones de sequía prolongada. Resultados similares fueron

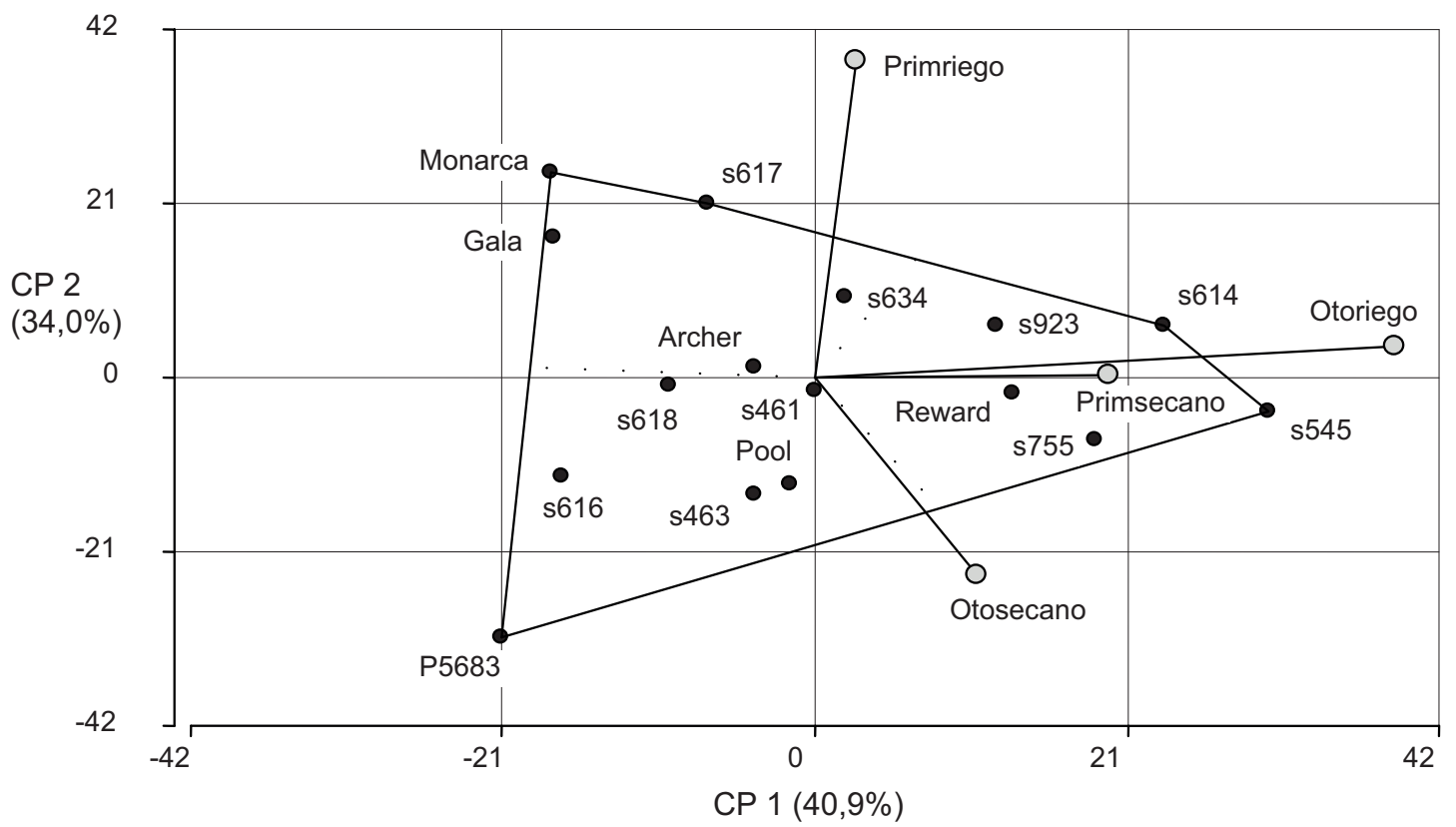

Figura 2. Plano biplot generado por el análisis GGE de la Cat 1 para la identificación de poblaciones y testigos en cada ambiente generado. 


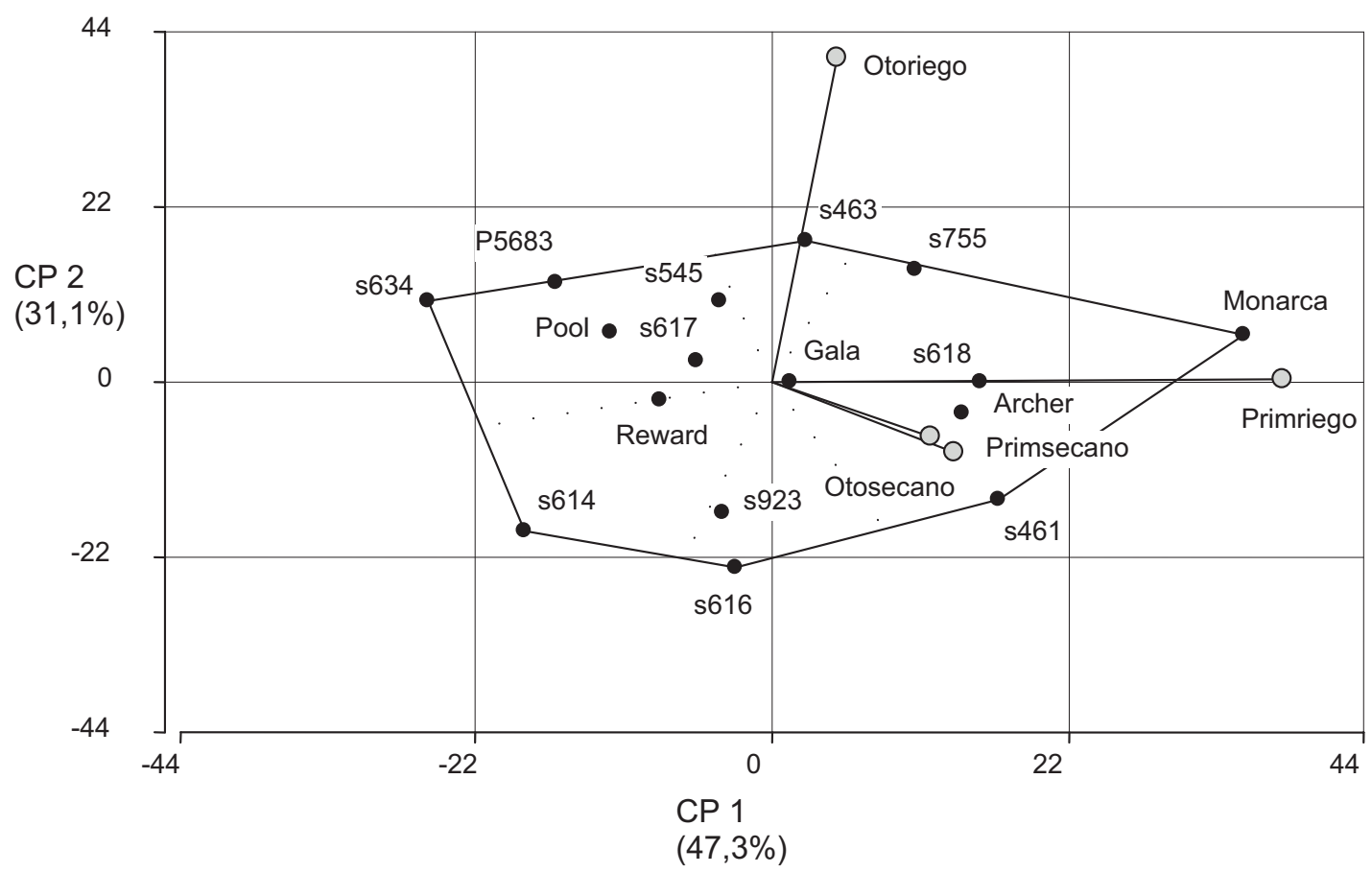

Figura 3. Plano biplot generado por el análisis GGE de la Cat 2+3 para la identificación de poblaciones y testigos en cada ambiente generado.

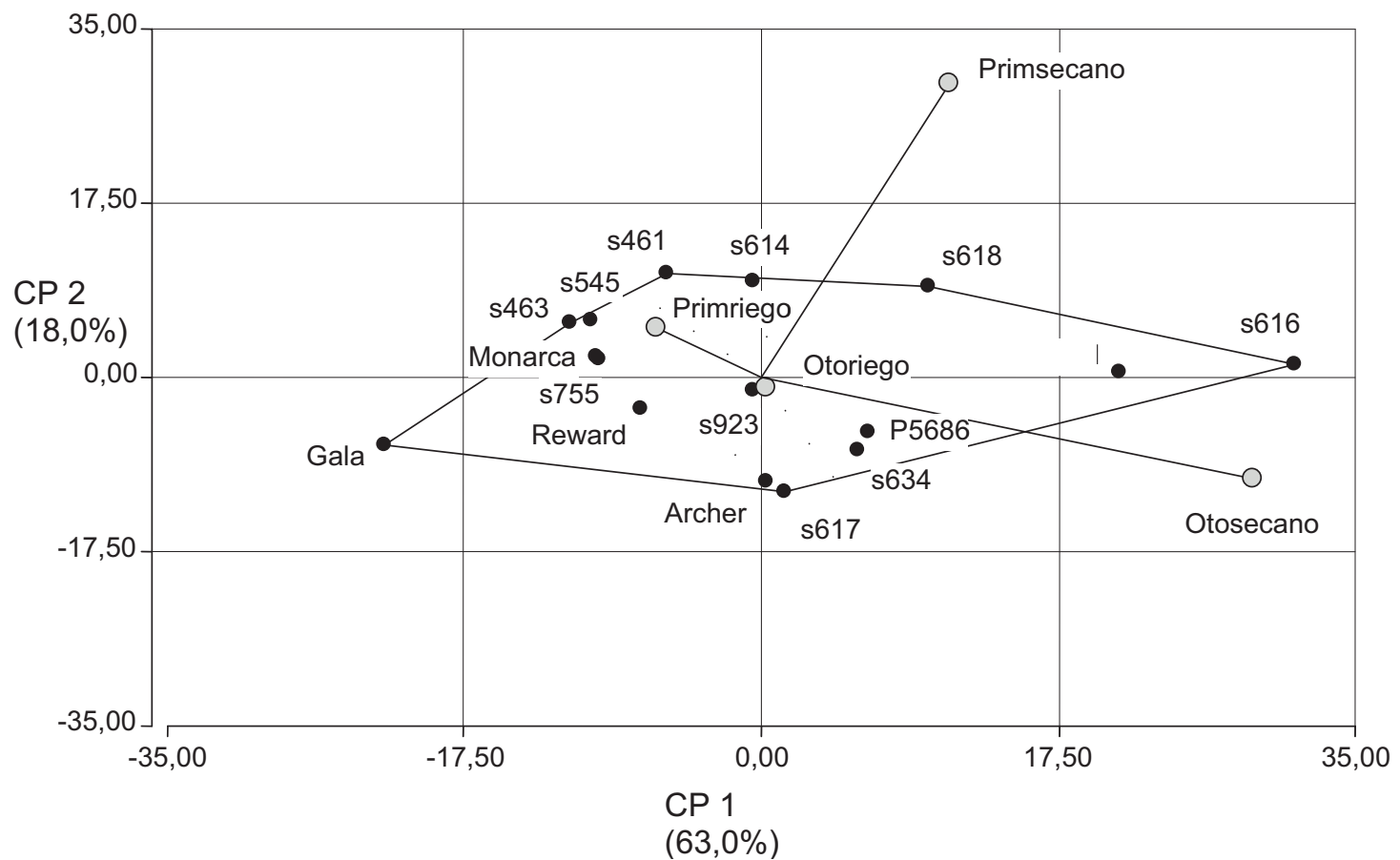

Figura 4. Plano biplot generado por el análisis GGE de la Cat 4+5 para la identificación de poblaciones y testigos en cada ambiente generado. 
informados por Gassen (1993) y Brewer (1976).

El análisis de la matriz de correlación fenotípica (r) (Tabla 2) indicó que: a) hubo más valores significativos en ambientes en secano que con riego, y que dentro de estos últimos, aumentaron en la primavera; b) rNRLR no presentó correlación con daño de gorgojos, pero se correlacionó positiva y significativamente con DRLR en todos los ambientes; c) DRLR se correlacionó positiva y significativamente con Cat $1, \mathrm{BP}$ y rNRLR, y negativa y significativamente con Cat $2+3$ y Cat $4+5$, pero sólo en el ambiente de primavera en secano; y d) BP sólo se correlacionó positivamente con la Cat 1 ( $\sin$ daño) en la siembra de primavera en secano y negativamente con daño severo (Cat $4+5)$ en los dos ambientes en secano (primavera y otoño). De esta forma, puede inferirse que bajos condiciones de deficiencia hídrica el mayor daño de gorgojos se corresponde con menor producción de forraje. Según Aragón (1991), esta situación es esperable ya que las plantas que tienen sus raíces afectadas por larvas de gorgojos disminuyen notoriamente su capacidad para extraer agua del suelo. Por otro lado, Romero (2004) comparó en condiciones de secano el comportamiento de dos cultivares de raíz ramificada con un testigo pivotante, y concluyó que la mayor expresión del carácter raíz ramificada y el menor daño de gorgojos de los dos primeros no se tradujeron en mayores rendimientos de biomasa aérea.

Es importante tener en cuenta que los resultados que se presentan en este trabajo corresponden sólo a una temporada de evaluación y que sería recomendable estudiar la evolución de los caracteres en temporadas posteriores, particularmente la correlación entre el NRLR, el daño de gorgojos y la producción de biomasa aérea, tanto en secano como en regadío. De igual forma, sería interesante determinar en la segunda y/o tercera temporada del cultivo la correlación entre DRLR y NRLR, dado que la mortandad de plantas que pudiera producirse en el futuro (sea por daño de gorgojos o por otros motivos) podría alterar la relación entre estos caracteres. En este sentido, se observó en el presente trabajo que aquellas plantas que dispusieron de mayor espacio entre ellas, o las que estaban sembradas en los bordes de las parcelas, presentaron no sólo raíces pivotantes más largas y de mayor diámetro, sino también mayor cantidad de raíces laterales. Esto último coincide con lo señalado por otros autores (Johnson et al., 1996; Garver, 1946; Upchurch y Lovvorn, 1951).

\section{BIBLIOGRAFÍA}

Altier, N. y R. Alzugaray, 1990. Incidencia de enfermedades y plagas en la producción y persistencia de alfalfa. En: Estación Experimental La Estanzuela. p.10.

Aragón, J. y J. Imwinkelried, 2007. Manejo integrado de plagas de la alfalfa. En: El cultivo de la alfalfa en la Argentina. D. H. Basigalup (Ed.) Buenos Aires: INTA, Cap. 9, pp. 165-197.

Aragón, J., 1990. Manejo integrado de plagas. Alfalfa. En: Cuaderno de actualización técnica N 49, CREA: 56-73.

Aragón, J., 1991. Desarrollo e implementación de un sistema de alarma con trampas de luz para orugas

Tabla 2. Correlaciones fenotípicas de Pearson por ambiente para las variables de raíz, biomasa aérea y daño entre poblaciones.

\begin{tabular}{|c|c|c|c|c|c|c|c|c|c|c|}
\hline \multicolumn{6}{|c|}{ Otoño secano } & \multicolumn{5}{|c|}{ Otoño riego } \\
\hline Variables $^{(\dagger)}$ & Cat 1 & $\begin{array}{l}\text { Cat } \\
2+3\end{array}$ & $\begin{array}{l}\text { Cat } \\
4+5\end{array}$ & $\mathrm{BP}$ & rNRLR & Cat 1 & $\begin{array}{l}\text { Cat } \\
2+3\end{array}$ & $\begin{array}{l}\text { Cat } \\
4+5\end{array}$ & $\mathrm{BP}$ & rNRLR \\
\hline Cat $2+3$ & $-0,27^{\star}$ & & & & & ns & & & & \\
\hline Cat $4+5$ & $-0,65^{\star *}$ & $-0,48^{*}$ & & & & $-0,34^{*}$ & $-0,48^{* *}$ & & & \\
\hline BP & ns & ns & $-0,35^{*}$ & & & ns & ns & ns & & \\
\hline rNRLR & ns & ns & ns & ns & & ns & ns & ns & ns & \\
\hline DRLR & ns & ns & ns & ns & $0,60^{* *}$ & ns & ns & ns & ns & $0,56^{\star \star}$ \\
\hline \multicolumn{6}{|c|}{ Primavera secano } & \multicolumn{5}{|c|}{ Primavera Riego } \\
\hline Cat $2+3$ & $-0,94^{\star *}$ & & & & & $-0,88^{\star \star}$ & & & & \\
\hline Cat $4+5$ & $-0,73^{\star \star}$ & $-0,45^{\star *}$ & & & & $-0,67^{\star *}$ & ns & & & \\
\hline BP & $0,66^{\star *}$ & ns & $-0,60^{* *}$ & & & ns & ns & ns & & \\
\hline rNRLR & ns & ns & ns & ns & & ns & ns & ns & ns & \\
\hline DRLR & $0,45^{\star *}$ & $-0,50^{* *}$ & $-0,41^{*}$ & $0,41^{*}$ & $0,76^{\star *}$ & ns & ns & $\mathrm{ns}$ & $\mathrm{ns}$ & $0,38^{*}$ \\
\hline
\end{tabular}

${ }^{(+)}$Cat: categoría de daño de gorgojos, BP: biomasa promedio, DRLR: diámetro de raíces laterales o ramificadas y rNRLR: raíz del número de raíces laterales o ramificadas.

*,**,ns: significativo a los niveles de probabilidad 0,05, 0,01 y no significativo, respectivamente. 
cortadoras y desfoliadoras. Proyecto PAN. INTA Marcos Juárez.

Balzarini, M. y J. Di Rienzo. 2004. Info-Gen: Software para análisis estadístico de datos genéticos. Universidad Nacional de Córdoba. Córdoba. Argentina.

Basigalup, D. y E. Hijano. 1995. Mejoramiento genético de la alfalfa. En: La alfalfa en la Argentina. Subprograma alfalfa. INTA Manfredi, .272 pp.

Bariggi, C., B. Marble, C. Itria y J. Brun (Eds.), 1986. Investigación, tecnología y producción de alfalfa. Colección científica del INTA, Tomo XXII, Buenos Aires, $488 \mathrm{pp}$.

Berry, P., 1947. Investigations on the white-fringed beetle groups in South America. J. Econ. Ent. 40(5): 705-709.

Brewer, M., 1976. Gorgojos de la alfalfa en la región semiárida de la Argentina. Generalidades, comportamiento en el laboratorio con dieta artificial y curvas poblacionales de adultos recogidos en diferentes trampas. Rev. Bolsa de Cereales (Buenos Aires) 2903: 3-15.

De Durana, J., 1981. Establecimiento y manejo del cultivo de alfalfa en la región subhúmeda y semiárida pampeana. Boletín de divulgación. № 3 INTA Anguil. La Pampa. 68 pp.

Dudley, J., R. Hill (Jr) and C. Hanson. 1963. Effects of seven cycles of recurrent phenotypic selection on means and genetic variances of several characters in two pools of alfalfa germplasm. Crop. Sci. 3: 543-546.

Garver, S., 1946. Alfalfa in South Dakota. SD. Agric. Expt. Stn. Bull. 383. 79 pp.

Gassen, D., 1993. Insetos de solo associados ao plantio direto. En: II Congreso Nacional de Siembra Directa, AAPRESID. Huerta Grande, Córdoba. pp. 46-49.

Gassen, D., 1996. Estratégias de manejo de pragas sobre plantío direto. En: IV Congreso Nacional de Siembra Directa, AAPRESID. Villa Giardino, Córdoba. pp. 253-278.

Harcourt, D., J. Aragón y R. González, 1986. Plagas de la alfalfa. En: Bariggi, C., B.

Marble, B., C. Itria y J. Brun (Eds.) Investigación, tecnología y producción de alfalfa. Colección científica del INTA, Tomo XXII, Buenos Aires, 488 pp.

Imwinkelried, J., R. Albrecht, C. Salto, R. Zheuder y A. Galetto, 1992. Implementación de una estrategia para el control integrado de plagas de la alfalfa en un área restringida de la provincia de Santa Fe. INTA-E.E.A. Rafaela (Arg.) Agronomía Inf. para Ext. № 151, 4 pp.

Itria, C., 1969. La alfalfa en la R. Argentina. I: Factores que disminuyen el rendimiento y duración de los cultivos. II: Contribución a la bibliografía nacional sobre alfalfa. IDIA Supl. 21.

Johnson, L., J. Marquez-Ortiz, D. Barnes and J. Lamb. 1996. Inheritance of root traits in alfalfa. Crop. Sci. 36: 1482-1487.

Johnson, L., J. Marquez-Ortiz, J. Lamb y D. Barnes. 1998. Root morphology of alfalfa plant introductions and cultivars. Crop Sci. 38: 497-502.

Odorizzi, A., D. Basigalup, V. Arolfo y M. Balzarini, 2008. Análisis de la variabilidad de caracteres de raíz en poblaciones de alfalfa (Medicago sativa L.) con alto número de raíces laterales. Agriscientia, Vol. XXV (2): 65-73.

Parodi, L., 1964. Enciclopedia argentina de agricultura y jardinería. Ed. Acme, Buenos Aires, 2 (2): 775 pp.

Romero, N., 2004. Evaluación a campo de variedades de alfalfas con raíz ramificada. En: Investigación en producción animal 2002-2003. Región subhúmeda y semiárida pampeana. Bol. Div. Téc. N 79. p. 45-54.

Sulc, R. y L. Rodes. 1997. Planting date, fungicide and cultivars effect on Sclerotinia crown and stem rot severity in alfalfa. Plant. Dist 81: 13-17.

Thomason, I., A. Gutiérrez y J. Lyons. 1985. Integrated pest management for alfalfa. Univ. of California, Div. Agric. Nat. Res. Publication 3312(4104), 96 pp.

Todd, J., 1968. Laboratory evaluation of insecticides against white-fringed weevil (Graphognatus leucoloma). J. Agric. Research. 11: 903-910.

Upchurch, R. y R. Lovvorn. 1951. Gross morphological root habits of alfalfa in North Carolina. Agron. J. 43:493-499. 